\title{
A 31P-Magnetic Resonance Spectroscopy investigation of peripheral skeletal muscle impairment in children after treatment for leukemia and lymphoma
}

Gillian White ${ }^{1}$, Sarah West ${ }^{2}$, Catherine Sabiston ${ }^{1}$, Shawn Rhind ${ }^{3}$, Paul Nathan ${ }^{4}$, Heather Jones $^{5}$, Tammy Rayner ${ }^{4}$, Ruth Weiss ${ }^{4}$, and Greg Wells ${ }^{4}$

${ }^{1}$ University of Toronto

${ }^{2}$ Trent University

${ }^{3}$ Defence Research and Development Canada Toronto Research Centre

${ }^{4}$ The Hospital for Sick Children

${ }^{5}$ Hospital for Sick Children

October 27, 2020

\begin{abstract}
Background: Exercise intolerance is a common side-effect of treatment for childhood leukemia and lymphoma and contributes to adverse health and well-being during survivorship. While central, cardiovascular contributors to fitness have been shown to be impaired, the peripheral muscular factors have not been studied in this population. Therefore, peripheral muscular function in children after leukemia and lymphoma treatment remains unstudied. Procedure: Eleven leukemia and lymphoma patients aged 8-18 years old who completed treatment 6-36 months prior and 11 healthy controls were included in analysis. $31 \mathrm{P}-\mathrm{MRS}$ was used to characterize muscle bioenergetic metabolism at rest and after in-magnet knee extension exercise. General exercise capacity was assessed using a submaximal graded treadmill test and overall physical activity participation was assessed using the Habitual Activity Estimation Scale (HAES). Results: The patients treated for leukemia and lymphoma exhibited lower anaerobic function $(\mathrm{d}=0.72)$, slower metabolic recovery $(\mathrm{d}=0.93)$, and lower mechanical muscle power $(\mathrm{d}=1.09)$ during in-magnet knee extension exercise compared with the healthy control group. Lower estimated VO2peak (41.61 \pm 5.97 vs. 47.71 \pm 9.99 ml.min-1.kg-1, $\mathrm{d}=0.76)$, lower self-reported minutes of physical activity $(58.3 \pm 35.3$ vs. $114.8 \pm 79.3$ minutes, $\mathrm{d}=0.99)$ and higher minutes of inactivity $(107.3 \pm 74.0$ vs. $43.5 \pm 48.3$ minutes, $\mathrm{d}=1.04, \mathrm{p}<0.05)$ were also observed in the patient group. Conclusions: Children treated for leukemia and lymphoma exhibit altered peripheral skeletal muscle energy metabolism in addition to previously reported central cardiovascular limitations during exercise. It is likely that both deconditioning and direct effects of chemotherapy treatment contribute to exercise intolerance in this population.
\end{abstract}

\section{Hosted file}

Manuscript - A 31P-Magnetic Resonance Spectroscopy investigation of peripheral skeletal muscle impairme available at https://authorea.com/users/370427/articles/489096-a-31p-magnetic-resonancespectroscopy-investigation-of-peripheral-skeletal-muscle-impairment-in-children-aftertreatment-for-leukemia-and-lymphoma 